\title{
Probing the Chiral Anomaly with Nonlocal Transport in Three-Dimensional Topological Semimetals
}

\author{
S. A. Parameswaran, ${ }^{1,2}$ T. Grover, ${ }^{3}$ D. A. Abanin, ${ }^{4,5}$ D. A. Pesin,,${ }^{6,7}$ and A. Vishwanath ${ }^{1,8}$ \\ ${ }^{1}$ Department of Physics, University of California, Berkeley, California 94720, USA \\ ${ }^{2}$ Department of Physics and Astronomy, University of California, Irvine, California 92697, USA \\ ${ }^{3}$ Kavli Institute for Theoretical Physics, University of California, Santa Barbara, California 93106, USA \\ ${ }^{4}$ Perimeter Institute for Theoretical Physics, Waterloo, Ontario N2L 2Y5, Canada \\ ${ }^{5}$ Institute for Quantum Computing, Waterloo, Ontario N2L 3G1, Canada \\ ${ }^{6}$ Department of Physics and Astronomy, University of Utah, Salt Lake City, Utah 84112, USA \\ ${ }^{7}$ Department of Physics, California Institute of Technology, Pasadena, California 91125, USA \\ ${ }^{8}$ Materials Science Division, Lawrence Berkeley National Laboratories, Berkeley, California 94720, USA \\ (Received 14 September 2013; revised manuscript received 4 March 2014; published 2 September 2014) \\ Weyl semimetals are three-dimensional crystalline systems where pairs of bands touch at points in \\ momentum space, termed Weyl nodes, that are characterized by a definite topological charge: the chirality. \\ Consequently, they exhibit the Adler-Bell-Jackiw anomaly, which in this condensed-matter realization \\ implies that the application of parallel electric $(\mathbf{E})$ and magnetic $(\mathbf{B})$ fields pumps electrons between nodes of \\ opposite chirality at a rate proportional to $\mathbf{E} \cdot \mathbf{B}$. We argue that this pumping is measurable via nonlocal \\ transport experiments, in the limit of weak internode scattering. Specifically, we show that as a consequence \\ of the anomaly, applying a local magnetic field parallel to an injected current induces a valley imbalance that \\ diffuses over long distances. A probe magnetic field can then convert this imbalance into a measurable \\ voltage drop far from source and drain. Such nonlocal transport vanishes when the injected current and \\ magnetic field are orthogonal and therefore serves as a test of the chiral anomaly. We further demonstrate \\ that a similar effect should also characterize Dirac semimetals - recently reported to have been observed in \\ experiments-where the coexistence of a pair of Weyl nodes at a single point in the Brillouin zone is \\ protected by a crystal symmetry. Since the nodes are analogous to valley degrees of freedom in \\ semiconductors, the existence of the anomaly suggests that valley currents in three-dimensional topological \\ semimetals can be controlled using electric fields, which has potential practical "valleytronic" applications.
}

DOI: 10.1103/PhysRevX.4.031035

\section{INTRODUCTION}

Weyl semimetals (WSMs) are three-dimensional analogs of graphene that have received much attention following a recent proposal that they may occur in a class of iridate materials [1]. They host electronic excitations that disperse linearly from degeneracy points at which two energy bands meet. Near these points, the electronic states are described by the Weyl equation, familiar from particle physics [1-4], and possess a definite chirality. While the robustness of such twofold band touchings-which require the breaking of either time-reversal or inversion symmetry-has long been known [2,3], the topological aspects of WSMs were only appreciated more recently $[1,4-6]$. A Weyl node is a topological object: Depending on its chirality, it acts as a source or sink of Chern flux in the Brillouin zone. Since the total Chern flux through the Brillouin zone must vanish,

Published by the American Physical Society under the terms of the Creative Commons Attribution 3.0 License. Further distribution of this work must maintain attribution to the author(s) and the published article's title, journal citation, and DOI.
Subject Areas: Condensed Matter Physics

Weyl nodes necessarily occur in pairs of opposite chirality. This topological property of the nodes protects a single Weyl node against opening a gap: In order to remove a Weyl band touching, a perturbation must necessarily couple the nodes, and thus WSMs should be robust against smooth disorder that only weakly mixes nodes separated in momentum space.

Closely related to the WSM is the Dirac semimetal (DSM) [7-9], where a pair of Weyl nodes of opposite chirality coexists at a point in the three-dimensional Brillouin zoneand therefore four bands touch, rather than two. Although, naively, it appears that this situation would be unstable against a variety of gap-opening scenarios, in certain cases, the resulting gapped phases always break a crystalline pointgroup symmetry. Therefore, as long as such symmetries are preserved, the Dirac point [10] remains stable. A convenient picture of the simplest DSM is two copies of a WSM, with each copy labeled by a different crystalline point-group "isospin" index. (To avoid confusion, we refer to the valley degree of freedom common to both cases as "pseudospin.") DSMs are thus crystalline symmetry-protected topological semimetals, and from the preceding discussion, it should be evident that stable three-dimensional Dirac points appear in 
pairs that lie on axes of high crystal symmetry. Recent photoemission [11-14] and magnetotransport [15] measurements appear to support the theoretical prediction $[8,9]$ that the three-dimensional materials $\mathrm{Na}_{3} \mathrm{Bi}$ and $\mathrm{Cd}_{3} \mathrm{As}_{2}$ host DSM phases.

Such robust topological phases are typically characterized by the presence of protected surface states or by unusual electromagnetic (EM) responses. WSMs are no exception: For instance, in Ref. [1], it was demonstrated that they possess protected chiral Fermi-arc surface states. Similar features are also expected for DSMs, as long as the protecting crystal symmetry remains unbroken; the resulting surface states, of course, now carry additional isospin labels.

The unconventional bulk EM response of a single threedimensional Weyl node is known as the Adler-Bell-Jackiw anomaly [5,16,17]: Simultaneous application of parallel electric and magnetic fields (applying $\mathbf{E} \cdot \mathbf{B}$ ) leads to production or depletion of charge, depending on the chirality. Such a response is clearly incompatible with charge conservation. The appearance of a Weyl node of opposite chirality resolves this apparent contradiction, since the charge produced (depleted) at one node is accounted for by that depleted (produced) at the other, and it is clear that the total charge is conserved. However, treating the node index (hereafter, "valley," in accord with the usual semiconductor terminology) as another quantum number, it is equally clear that the valley charge is not conserved in the presence of $\mathbf{E} \cdot \mathbf{B}$ : This is the chiral anomaly of the WSM. For the DSM, as we have mentioned, in addition to the valley pseudospin index that labels the point in the Brillouin zones where the bands touch, there is an additional twofold isospin index that labels the crystalline point-group representation. The discussion of the anomaly goes through more or less unchanged for each of these isospin species.

How can we observe this effect in experiments?

Any proposal to detect the anomaly should be capable of distinguishing anomaly-related physics from conventional metallic behavior and should ideally vanish in the absence of the anomaly-i.e., either when $\mathbf{E} \cdot \mathbf{B}=0$ or in the absence of Weyl nodes; furthermore, it should be applicable to topological semimetals realized in several different systems - in other words, we seek a response characteristic of the phase rather than of any specific realization.

Here, we show that the slow relaxation of valley charge (characterized by an internode scattering time $\tau_{v}$, which is typically long, as it involves large quasimomentum transfer in the Weyl case or scattering between different point-group representations in the Dirac case) results in a signature of the charge pumping in nonlocal resistance measurements (Fig. 1). While, in general, sensitive to various experimental parameters, in the "quantum" limit, when the valley imbalance generated is limited only by relaxation at the contacts, we find that applying a voltage $V_{\mathrm{SD}}$ at $x=0$

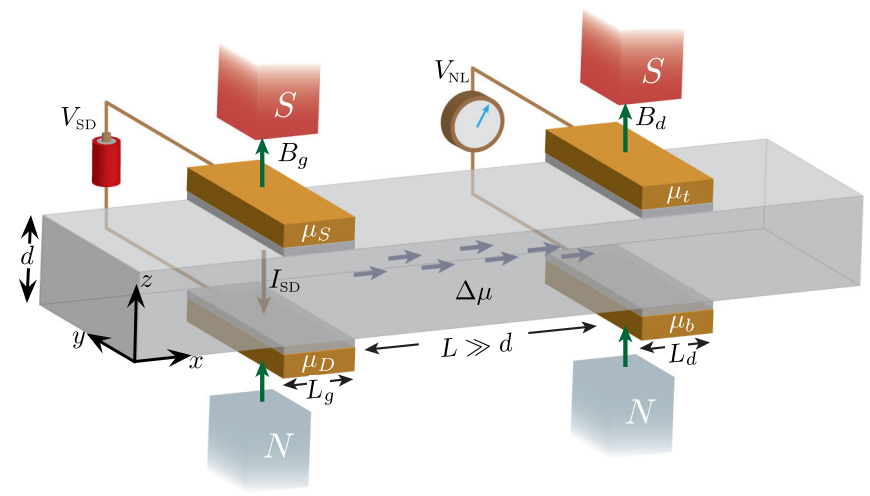

FIG. 1. Nonlocal transport experiment. A source-drain current $I_{\mathrm{SD}}$ is injected into a Weyl semimetal slab of thickness $d$ via tunneling contacts of thickness $L_{g}$. In the presence of a local generation magnetic field $B_{g}$, a valley imbalance $\Delta \mu$ is created via the chiral anomaly and diffuses a distance $L \gg d$ away. If a detection field $B_{d}$ is applied, the valley imbalance can be converted into a potential difference $V_{\mathrm{NL}}$ between top and bottom contacts of size $L_{d}$.

yields a nonlocal voltage at $x$ that is determined only by intervalley relaxation in the bulk,

$$
\left|V_{\mathrm{NL}}(x)\right|=V_{\mathrm{SD}} e^{-x / \ell_{v}},
$$

where $\ell_{v}=\sqrt{D \tau_{v}}$ is the valley-relaxation length and $D$ is the charge-diffusion coefficient. In contrast, conventional Ohmic voltages decay on the scale of the sample thickness. In addition, the dependence of nonlocal response on field orientation (described in detail below) further reflects its origins in the anomaly. In particular, $V_{\mathrm{NL}}=0$ when an $\mathbf{E} \cdot \mathbf{B}$ term is absent. Thus, in the idealized limit $\ell_{v} \rightarrow \infty$, this nonlocal response gives an unambiguous signature of the anomaly.

The proposed experiment is easily sketched; for the moment, let us focus on the Weyl case. First, a charge current is driven across the sample in a region where a local magnetic field $B_{g}$ is applied. Because of the chiral anomaly, in the steady state, a valley imbalance-in the ideal case, proportional to $B_{g}$-is generated in the region where charge current flows. As long as the internode scattering is weak, the valley imbalance is long lived and can diffuse far away (i.e., a distance of $\operatorname{order} \ell_{v}$ ) from the region where it is generated. In the absence of a magnetic field, the valley imbalance does not couple to an electric field and is thus challenging to detect. However, such a coupling does arise when a local "detection" magnetic field $B_{d}$ is applied-once again, a consequence of the chiral anomaly. In this case, the valley imbalance manifests itself by building up an electrical voltage across the sample. When $B_{g}$ is oriented perpendicular to the sourcedrain current, or $B_{d}$ perpendicular to the direction in which the voltage drop is measured, $V_{\mathrm{NL}}$ vanishes, reflecting the fact that the anomaly is sensitive to the angle between $\mathbf{E}$ and $\mathbf{B}$. While this nonlocal effect bears some resemblance to the socalled "Zeeman-driven spin Hall effect" and associated 
transport phenomena in graphene [18-21] and other spin Hall materials, the dependence on the orientation of the magnetic field is unique and is a signature of the chiral anomaly.

Before proceeding, we briefly review other recent proposals to study the anomaly in WSMs (We note that similar anomalies have been previously explored in experiments on vortices in superfluid ${ }^{3} \mathrm{He}$ [22].) One observation [5] is that there is an additional, anomaly-induced current along the magnetic field direction; the resulting anisotropy in the magnetoconductance has been suggested $[23,24]$ as a signature of the anomaly. However, one might expect such anisotropy simply on symmetry grounds [25] in a conventional metal, since the magnetic field provides a preferred direction. Additionally, the anomaly results in a negative classical magnetoresistance that can be quite large [24]; however, this remains a quantitative rather than a qualitative signature and may be overwhelmed by other contributions, making it challenging to detect. Another proposal [26] is to realize a WSM by magnetically doping a topological insulator; the symmetry-breaking ferromagnetic order can then be used as a probe of the underlying topological semimetal. For instance, as a consequence of the anomaly, vortex lines in the ferromagnet carry one-dimensional chiral modes, and the ferromagnetic Goldstone modes couple to charge plasmons. While striking, such features are not easy to probe and are specific to the example studied rather than serving as a general signature of a WSM. In Ref. [27], an anomalous Hall-effect signature was discussed, related to the Weyl anomaly [6]; however, this is absent for certain highsymmetry crystals and needs additional information on the momentum-space location of nodes to be turned into a sharp signature. Thus, existing approaches to study the topological response of WSMs stand in marked contrast to the simple transport experiment proposed here, which applies generally to all realizations of WSMs and furthermore satisfies the criteria outlined earlier: Namely, it involves a signal that is absent for conventional (semi)metals and can be ascribed to the presence of an $\mathbf{E} \cdot \mathbf{B}$ term by examining its dependence on the orientation of the magnetic field. Furthermore, as we demonstrate, modulo some reasonable caveats about disorder, our results also apply to DSMs. We note that, in contrast to other transport-related predictions, our proposal involves a physical mechanism - the diffusion of valley imbalancethat is distinct from the transport of electric charge and is crucial to the nonlocality of the response. As a consequence of the nonlocality, we may attribute our signal to a specific magnetoresistance mechanism (namely, the anomaly) circumventing the challenges usually involved in interpreting magnetotransport measurements. The experiment proposed here thus serves as an "order parameter" for topological semimetals of both the Dirac and Weyl varieties and is the only sharp signature proposed to date that is agnostic to the specific details of the experimental realization.

In the remainder, we first outline a simple description of transport in a WSM and proceed to discuss the chiral charge pumping within this formalism. Having formulated a limit where the solution is especially transparent, we demonstrate the existence of a nonlocal response and examine its behavior in various cases before turning to a simple model of impurity scattering that permits us to provide parametric estimates of various length scales; we also demonstrate that our results remain applicable to the case of a disordered DSM. We conclude with a discussion of our results and possible extensions.

\section{MODEL AND TRANSPORT THEORY FOR WEYL SEMIMETALS}

\section{A. Transport equations}

We begin by sketching the derivation of the transport equations relevant to the problem in the WSM case. The simplest models of WSMs have two nodes separated in momentum space, and henceforth, we specialize our discussion to this situation. (The extension of our results to the case with several such pairs of nodes is straightforward; we will say more about the DSM, where there are additional subtleties owing to the coincidence of two Weyl nodes at a single Dirac point, below.) Electrons emanating from the two valleys [denoted by "right" $(R)$ and "left" $(L)$ and referred to as pseudospin] are characterized by local electrochemical potentials in each valley

$$
\mu_{\mathrm{EC}}^{R, L}=\mu^{R, L}+e \phi,
$$

defined as the sum of the electric potential $\phi$ and the valley chemical potential $\mu^{R, L}$. We assume that each valley has the same finite doping level, so that the density of states $\nu_{3 \mathrm{D}}$ is finite and equal in both valleys. As a result, charge transport within a valley is characterized (at $B=0$ ) by a finite Drude conductivity $\sigma$ related to the diffusion coefficient $D$ via the Einstein relation $\sigma=e^{2} D \nu_{3 \mathrm{D}}$. All the chemical potentials are measured with respect to thermal equilibrium, so that the expressions below do not include any equilibrium "magnetization" currents [28].

In a magnetic field $\mathbf{B} \equiv B \hat{\mathbf{n}}$, the currents in each valley can be expressed purely in terms of the potentials by solving for the Landau levels (LLs) of the Weyl nodes. Recall that a single node [29] gives rise to an infinite set of LLs that disperse quadratically in the field direction $E_{n}^{R, L}(\mathbf{k} \cdot \hat{\mathbf{n}})=\hbar v_{F} \operatorname{sgn}(n) \sqrt{2|n| e B / \hbar c+(\mathbf{k} \cdot \hat{\mathbf{n}})^{2}}, \quad$ with $n= \pm 1, \pm 2, \ldots$, as well as a single $(n=0)$ LL with $E_{0}^{R, L}(\mathbf{k} \cdot \hat{\mathbf{n}})= \pm \hbar v_{F} \mathbf{k} \cdot \hat{\mathbf{n}}$ that disperses linearly along the field, with a chirality set by that of the node (Fig. 2). Each energy level is degenerate, with $N_{\Phi} / A=$ $1 / 2 \pi \ell_{B}^{2}$ states per unit area, where $\ell_{B}=(\hbar c / e B)^{1 / 2}$ is the magnetic length corresponding to the magnetic field $B$. As a consequence of the chiral $n=0 \mathrm{LL}$, electrons at a Weyl node carry a current along the field even for a spatially uniform chemical potential; the total anomaly-related current is obtained by summing over all the occupied 


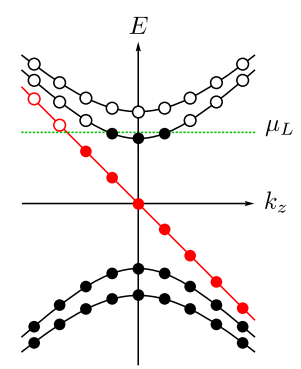

$L$

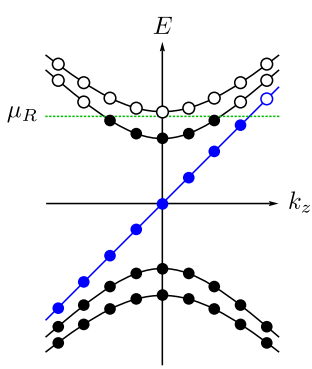

$R$
FIG. 2. Landau levels at Weyl nodes. Filled (empty) circles denote occupied (empty) LLs. Each node has nonchiral LLs that disperse parabolically in the field direction (here, $\hat{\mathbf{z}}$ ) as well as a single chiral LL that disperses according to the node chirality (red and blue circles and lines). A chemical potential imbalance between the nodes leads to a net current flowing along the field, even for spatially uniform $\mu$.

modes in this LL. In addition, in each valley, there is also the conventional transport contribution due to gradients of the electrochemical potential. In our semiclassical limit, we assume this contribution deviates only weakly from its zero-field value and is therefore well described in terms of the Drude conductivity $\sigma$. These two contributions combine to give the total transport current density [30]

$$
j^{R, L}=-\frac{\sigma}{e} \nabla \mu_{\mathrm{EC}}^{R, L} \pm \frac{e^{2} \mathbf{B}}{4 \pi^{2} \hbar^{2} c} \mu^{R, L} .
$$

These equations should be complemented by the continuity equations. In the presence of the chiral anomaly, we have [31]

$$
\nabla \cdot j^{R, L}+\partial_{t} \rho^{R, L}= \pm \frac{e^{3}}{4 \pi^{2} \hbar^{2} c} \mathbf{E} \cdot \mathbf{B},
$$

where the $\mathbf{E} \cdot \mathbf{B}=-\mathbf{B} \cdot \nabla \phi$ term $[5,16,17]$ is due to the anomaly and captures the valley charge pumping. Using Eq. (2) and modeling intervalley relaxation via a characteristic scattering rate by impurities $1 / \tau_{v}$, $\partial_{t} \rho^{R, L}= \pm\left(1 / 2 \tau_{v}\right)\left(\rho^{R}-\rho^{L}\right)$, the steady-state continuity equations in the two valleys reduce to

$$
-\frac{\sigma}{e} \nabla^{2} \mu_{\mathrm{EC}}^{R, L} \pm \frac{\beta}{e} \hat{\mathbf{n}} \cdot \nabla \mu_{\mathrm{EC}}^{R, L}=\mp \frac{e \nu_{3 \mathrm{D}}}{2 \tau_{v}}\left(\mu_{\mathrm{EC}}^{R}-\mu_{\mathrm{EC}}^{L}\right),
$$

where $\beta=\left(1 / 2 \pi \ell_{B}^{2}\right)\left(e^{2} / h\right)$. Note that the continuity equations depend only on the electrochemical potential, unlike the currents.

Equations (2) and (4) supplemented by appropriate boundary conditions determine the charge and valley currents in the system. From this point on, we specialize to the setup illustrated in Fig. 1 and choose coordinates in which the $z$ direction is perpendicular to the film.

\section{B. Boundary conditions}

We now establish the current boundary conditions in the presence of leads and magnetic field. We assume that the boundaries do not induce intervalley scattering [32]. The boundary conditions become especially transparent when treating the interfaces in the Landauer formalism (see Fig. 3) and with the assumption of no intervalley scattering in the leads. Let us assume that there are $N_{n}$ nonchiral channels in a region of area $A$ [33], each with transmission coefficient $\mathcal{T}_{i}$. Within the Landauer picture, these channels carry a current that depends on the contact conductance per unit area $g=$ $\left(e^{2} / h\right)(1 / A) \sum_{i=1}^{N_{n}} \mathcal{T}_{i}$ and the electrochemical potential between the contact and the WSM surface. In contrast, the chiral channels in each node carry a current that depends only on the electrochemical potential on one side of the interfacei.e., either that of the contact or of the WSM, depending on the direction. With these considerations, and introducing source and drain chemical potentials $\mu_{S, D}$, we find that the boundary conditions for the top surface are

$$
\begin{aligned}
& j_{z}^{R}(d)=\frac{g}{e}\left[\mu_{\mathrm{EC}}^{R}(d)-\mu_{S}\right]+\frac{\beta}{e} \mu_{\mathrm{EC}}^{R}(d), \\
& j_{z}^{L}(d)=\frac{g}{e}\left[\mu_{\mathrm{EC}}^{L}(d)-\mu_{S}\right]-\frac{\beta}{e} \mu_{S},
\end{aligned}
$$

while on the bottom surface, we have

$$
\begin{aligned}
& j_{z}^{R}(0)=\frac{g}{e}\left[\mu_{D}-\mu_{\mathrm{EC}}^{R}(0)\right]+\frac{\beta}{e} \mu_{D}, \\
& j_{z}^{L}(0)=\frac{g}{e}\left[\mu_{D}-\mu_{\mathrm{EC}}^{L}(0)\right]-\frac{\beta}{e} \mu_{\mathrm{EC}}^{L}(0) .
\end{aligned}
$$

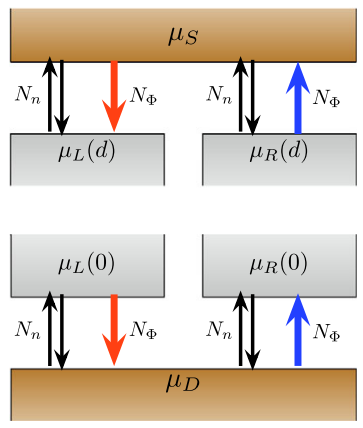

FIG. 3. Landauer description of contact boundary conditions. Each node has $N_{n} \gg 1$ nonchiral normal transport channels in an area $A$ (black arrows), with transmission coefficient $\mathcal{T}_{i}$. Since we assume that there is no intervalley scattering at the interfaces, we can simply count the number of channels and obtain a normal conductance per unit area $g=\left(e^{2} / h\right)(1 / A) \sum_{i=1}^{N_{n}} \mathcal{T}_{i}$ for these channels. Here, $\mathcal{T}_{i}$ is the transmission coefficient of channel $i$, assumed much smaller than unity for much of the paper (tunneling contacts). In addition, in each node, there are also $N_{\Phi}$ chiral channels propagating in opposite directions in the two nodes (red and blue arrows), where $N_{\Phi}=A / 2 \pi \ell_{B}^{2}$ is the number of flux quanta threading the contact area. Applying the Landauer formalism, assuming reflectionless contacts, and assigning appropriate chemical potentials to the different channels, we obtain boundary conditions (5) and (6) for the current density. 
In the above equations, it is understood that $\beta=$ $\operatorname{sgn}\left(B_{z}\right)\left(e^{2} / 2 \pi l_{B}^{2} h\right)$; we recognize this as the conductance per unit area of the chiral modes, which have $\mathcal{T}=1$. We will assume that in both generation and detection regions, $\mathbf{B}$ lies in the $y z$ plane, inclined at an angle $\theta$ from $\hat{\mathbf{z}}$. In this case, we have $\beta \propto \cos (\theta)=\mathbf{B} \cdot \hat{\mathbf{z}} / B$. Below, we will focus on the case when the field is along $\hat{\mathbf{z}}$, i.e., $\theta=0$.

\section{Relaxation in leads}

We digress briefly to discuss a subtlety that emerges when placing leads on a WSM. We wish to induce and detect disequilibrium between valley populations. However, a normal-metal lead attached to a WSM has, in effect, a vanishingly small relaxation length for the valley degree of freedom; ideal contacts to such leads therefore suppress valley imbalance [34]. Therefore, for a given conductivity of the WSM film, tunneling contacts to metallic leads [35] generally sustain a larger valley imbalance. For simplicity, we work in the thin-film limit $g \ll \sigma / d$, where $d$ is the thickness of the film. This condition implies that voltages are built up only across the surfaces, while inside the film, fast diffusion makes the electrochemical potentials uniform across the film thickness. However, it should be kept in mind that this assumption is not necessary for nonlocal transport to arise; we will discuss the case of transparent contacts below.

\section{NONLOCAL RESPONSE}

We will work in the thin-film limit, where the various chemical and electrochemical potentials are assumed uniform across the film thickness. In this situation, averaging the continuity equations over the film thickness and using the boundary conditions (5) and (6), we find

$$
\begin{aligned}
\nabla_{\perp}^{2} \mu_{\mathrm{EC}}^{\mathrm{av}} & =\frac{2 g+\beta}{\sigma d}\left(\mu_{\mathrm{EC}}^{\mathrm{av}}-\frac{\mu_{S}+\mu_{D}}{2}\right), \\
\nabla_{\perp}^{2} \delta \mu_{\mathrm{EC}} & =\frac{1}{\ell_{\mathrm{eff}}^{2}} \delta \mu_{\mathrm{EC}}+\frac{\beta}{\sigma d}\left(\mu_{S}-\mu_{D}\right),
\end{aligned}
$$

where $\nabla_{\perp}^{2}=\partial_{x}^{2}+\partial_{y}^{2}$. We have defined the average electrochemical potential $\mu_{\mathrm{EC}}^{\mathrm{av}}=(1 / 2 d) \int_{0}^{d} d z\left(\mu_{\mathrm{EC}}^{R}+\mu_{\mathrm{EC}}^{L}\right)$ and the difference between valley electrochemical potentials $\delta \mu_{\mathrm{EC}}=(1 / d) \int_{0}^{d} d z\left(\mu_{\mathrm{EC}}^{R}-\mu_{\mathrm{EC}}^{L}\right)$ and finally introduced the effective valley-imbalance-relaxation length

$$
\ell_{\text {eff }}^{-2}=\frac{1}{D \tau_{v}}+\frac{2 g+\beta}{\sigma d} .
$$

As discussed above, the leads induce additional intervalley relaxation, at a rate $\Gamma_{\text {leads }}=(2 g+\beta) / \nu_{3 \mathrm{D}} e^{2} d$.

Equations (7) allow us to analyze the valley transport in a thin WSM film in the presence of external leads. The generation region is taken to consist of two massive source and drain leads of width $L_{g} \gg \ell_{\text {eff }}$. In this limit, they act as a valley battery, inducing in the source-drain region a valley imbalance

$$
\delta \mu_{\mathrm{EC}}(0)=-\frac{\beta_{g} \ell_{\mathrm{eff}, g}^{2}}{\sigma d}\left(\mu_{S}-\mu_{D}\right)
$$

Here, the subscript $g$ labels parameters pertaining to the generation region and the corresponding local magnetic field $B_{g}$. Note that even though there is a negligible electrochemical potential drop across the film when $g \ll \sigma / d$, a valley imbalance is nevertheless generated by the preferential population of chiral modes at sample boundaries. This is essentially equivalent to the effect of the $\mathbf{E} \cdot \mathbf{B}$ term, with $\nabla \mu_{\mathrm{EC}}$ playing the role of the electric field.

The imbalance generated in the source-drain region diffuses over the sample, but due to intervalley scattering, the imbalance decays away from the generation region with a characteristic length $\ell_{v}=\sqrt{D \tau_{v}}$. This follows from the fact that in the region between the "battery" and "detector" leads, there are no contacts, and also, we have $\mathbf{B}=0$, under which conditions we assume that the surfaces do not induce intervalley scattering. Then, the propagation length of the valley imbalance is maximal and limited only by the weak intervalley impurity scattering $\ell_{\text {eff }}=\ell_{v}$, and so

$$
\delta \mu_{\mathrm{EC}}(x)=\delta \mu_{\mathrm{EC}}(0) e^{-\left(|x| / \ell_{v}\right)} .
$$

To detect this imbalance far away from the generation region, one can place voltage probes in a region where a local detector magnetic field $B_{d}$, with the corresponding $\beta_{d}$, is applied. The chiral anomaly will then transform valley imbalance into charge current in the vertical direction. In order to compensate this current, a measurable voltage drop is developed between the top and bottom detecting leads. We assume that the detector is a noninvasive probe; that is, it does not alter the value of the valley imbalance it measures. This imposes a restriction on the length of the detecting leads, which will be formulated below.

Demanding that the total current through the top and bottom contacts of the detector vanishes and using [Eqs. (5) and (6)] yields for the measured chemical potential difference between them,

$$
\mu_{t}-\mu_{b}=\frac{\beta_{d}}{2 g_{d}+\beta_{d}} \delta \mu_{\mathrm{EC}}(x) .
$$

Using Eqs. (9) and (10), we can relate the measured "nonlocal" voltage drop $V_{\mathrm{NL}}=\left(\mu_{t}-\mu_{b}\right) / e$ to the source-drain voltage $V_{\mathrm{SD}}=\left(\mu_{S}-\mu_{D}\right) / e$. It is convenient to introduce a dimensionless coefficient $\alpha_{\mathrm{NL}}$ that characterizes the strength of nonlocal response as a ratio of these voltages:

$$
\alpha_{\mathrm{NL}}(x)=\frac{V_{\mathrm{NL}}(x)}{V_{\mathrm{SD}}}=-\frac{\beta_{d}}{2 g_{d}+\beta_{d}} \frac{\beta_{g} \ell_{\mathrm{eff}, g}^{2}}{\sigma d} e^{-\left(|x| / \ell_{v}\right)} .
$$


This equation takes an even more transparent form if we assume that in the generation region, relaxation due to intervalley scattering can be completely neglected and occurs solely at the leads. In this case, we can neglect the first term in the lhs of Eq. (8) for $\ell_{\mathrm{eff}, g}$, which yields

$$
\alpha_{\mathrm{NL}}(x)=-\frac{\beta_{d}}{2 g_{d}+\beta_{d}} \frac{\beta_{g}}{2 g_{g}+\beta_{g}} e^{-\left(|x| / \ell_{v}\right)}
$$

This equation is the central result of the paper and gives the general dependence of the nonlocal transport on contacts, fields, and intervalley relaxation in the limit when the latter is weak. Note that this condition is not unreasonable: A WSM and its attendant topological features are stable only for weak intervalley scattering, corresponding to large $\ell_{v}$. Furthermore, it is in this limit-specifically, for $\ell_{v} \gg d-$ that the nonlocal response dominates standard Ohmic voltages between the film surfaces. It is instructive to analyze Eq. (13) in two limits. (i) In the limit of weak generation and detection magnetic field $\beta_{g} \ll g_{g}$ and $\beta_{d} \ll g_{d}$, the nonlocal response

$$
\alpha_{\mathrm{NL}}(x) \approx-\frac{\beta_{d}}{2 g_{d}} \frac{\beta_{g}}{2 g_{g}} e^{-\left(|x| / \ell_{v}\right)}
$$

is proportional to the magnetic fields $B_{g}$ and $B_{d}$ and changes sign if the direction of one of these fields is reversed. In this limit, the nonlocal voltage is inversely proportional to the conductance of the contacts $g_{g}$ and $g_{d}$. Therefore, the nonlocal voltage is larger for tunneling contacts, as long as they continue to have a higher conductance than the chiral channels. (ii) In the opposite limit, when $g_{d} \ll \beta_{d}$ and $g_{g} \ll \beta_{g}$, it takes a remarkably simple form $\alpha_{\mathrm{NL}}(x) \approx-e^{-\left(|x| / \ell_{v}\right)}$, equivalent to that quoted in Eq. (1), and depends neither on the properties of the contacts nor on the magnitude of the generating and detecting magnetic fields (although, of course, a directional dependence remains). This is the quantum limit, when the generation of valley imbalance is limited by the relaxation at the contact itself. Note that $\beta=\left(\lambda_{F} / \ell_{B}\right)^{2} e^{2} / 2 \pi \lambda_{F}^{2} h$, where $\lambda_{F}$ is the Fermi wave vector in the node and $e^{2} / h \lambda_{F}^{2} \propto g_{\text {ideal }}$ is the maximal (Sharvin) contact conductance per unit area. In order that the semiclassical limit holds, we wish to have many filled LLs below the Fermi surface, which assumes that $\lambda_{F} / \ell_{B}$ is small, requiring $\beta \ll g_{\text {ideal }}$. However, since tunneling contacts have $g \ll g_{\text {ideal }}$, this requirement is not too restrictive, and we expect that the quantum limit can indeed be reached in experiments.

Finally, we revisit our assumption that the detector does not alter the value of the valley imbalance it measures; this constrains detectorsize, as follows. The detector is essentially a shunt connecting two valleys, allowing valley current to "leak" at a rate $\Gamma_{\text {leads }}$. The detector is noninvasive if this leakage current is much smaller than the total valley current flowing under the detector $j_{v} \sim D \delta \mu / \ell_{v}$. Comparing the latter to leakage current $j_{\text {leak }} \sim \Gamma_{\text {leads }} L_{d} \delta \mu$, we obtain that the detector size $L_{d} \ll(\sigma / D) /\left(2 g_{d}+\beta_{d}\right) \times\left(d^{2} / \ell_{v}\right)$. This condition is quite weak, since $d \ll L_{d} \ll \ell_{v}$ can be satisfied for large enough $\sigma / d\left(2 g_{d}+\beta_{d}\right)$, which is well within the tunneling contact or thin-film limit discussed here.

\section{A. Extension to Dirac semimetals}

The situation in the case of a Dirac semimetal is slightly more involved; this is because at each Dirac point, there is a pair of Weyl nodes of opposite chirality distinguished by the point-group index or isospin. Thus, for each isospin, one obtains a scenario similar to that described above, with valley imbalance having an opposite sign for the two isospin species. In order for this simple picture to hold, we must make two crucial approximations. The first is to ignore higher-order terms in the dispersion, which lead to mixing of chiralities; the second is that we assume that the isospin-relaxation time $\tau_{i}$ due to impurity scattering is much larger than the valley-relaxation time, in order that we may treat isospin as a "good" quantum number over the length and time scales relevant to our experiment. We will discuss both these approximations in the next section. Note also that the relevant time scale for valley relaxation is the shorter of $\tau_{v}$ and $\tau_{i}$ since strong scattering between isospin species will relax the valley imbalance. This follows because in any situation, where, at a given valley, the population of one isospin species increases due to the anomaly, the population of the other isospin decreases, as the anomalous contribution has the opposite sign for the two. With this caveat, the rest of the argument goes through identically, and one obtains a similar nonlocal transport signature as Eqs. (1) and (12)-(14) with $\ell_{v}$ replaced by $\min \left(\sqrt{D \tau_{v}}, \sqrt{D \tau_{i}}\right)$. In the next section, we will discuss estimates for the relevant time scales using a simple disorder model.

\section{RELAXATION PROCESSES IN DISORDERED DIRAC OR WEYL SEMIMETALS}

\section{A. Charge and valley relaxation in WSM}

In this section, we use a perturbative treatment of disorder to estimate the characteristic rate of two scattering processes relevant to a WSM (see Fig. 4): (i) quasiparticle scattering at a single Weyl node, which relaxes charge imbalance at a rate $\tau_{c}^{-1}$ and (ii) intervalley (i.e., pseudospinflip) scattering, which relaxes valley imbalance at a rate $\tau_{v}^{-1}$. (We will discuss extensions to the Dirac case, where there is an additional issue of isospin relaxation, below.)

In order that we can distinguish the nonlocal and Ohmic responses, we require that $d \ll \sqrt{D \tau_{v}}$. Furthermore, to define a local diffusive charge conductivity $\sigma$ while treating the valley imbalance as a slowly relaxing quantity, we need $\tau_{c} \ll \tau_{v}$. When both these criteria are satisfied, the nonlocal response can be clearly distinguished and is then a measure of the anomaly in the WSM. In the DSM, we additionally 


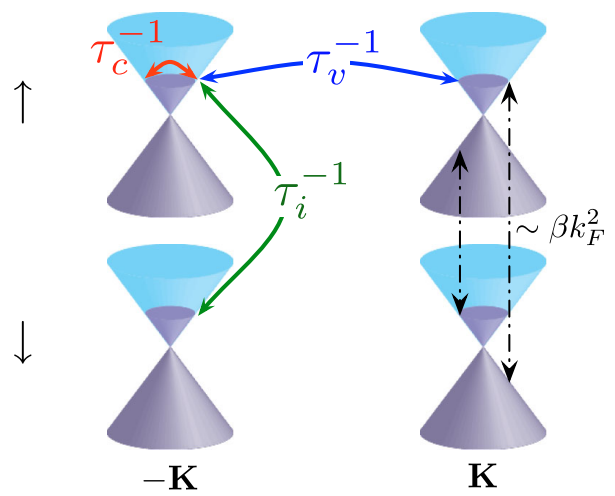

FIG. 4. Charge, valley, and isospin relaxation and mixing in topological semimetals. We depict the different relaxation processes in disordered topological semimetals. Charge is relaxed by processes that scatter within a single Weyl node $\left(\tau_{c}^{-1}\right)$; valley pseudospin (indicated by node position $\pm \mathbf{K}$ ) is relaxed by processes involving large momentum transfer, which for screened impurities leads to strong suppression relative to charge relaxation $\tau_{v}^{-1} \sim \tau_{c}^{-1}\left(k_{F} / 2 K\right)^{4}$; and in the Dirac case, the point-group isospin (indicated by $\uparrow$ and $\downarrow$ ) is relaxed by scattering between the two Weyl nodes at a single Dirac point, which are shown separately for clarity but are, in fact, degenerate. Finally, curvature terms in the Dirac case mix electronlike (holelike) isospin- $\uparrow$ states with holelike (electronlike) isospin- $\downarrow$ states with a strength of approximately $\beta k_{F}^{2}$. Note that, in fact, the isospin relaxation occurs by a combination of this mixing and charge relaxation at a single node, yielding $\tau_{i}^{-1} \sim \tau_{c}^{-1}\left(\beta k_{F}^{2} / \epsilon_{F}\right)^{2}$.

require that all length scales are small compared to $\sqrt{D \tau_{i}}$, so that our assumption of treating the two isospin species as independent is reasonable over scales at which we measure unambiguously nonlocal effects.

We consider scattering from impurities randomly distributed with average density $n_{\text {imp }}$, each of which we shall assume is modeled by a smooth central potential $v(r)$. We will assume that (in the absence of external fields) each node is doped so that the chemical potential is away from the nodal point; we therefore take the (equilibrium) Fermi level in each node to be $\epsilon_{F}=\hbar v_{F} k_{F}$. We shall assume furthermore that the Fourier transform of the impurity potential takes the form

$$
v(\mathbf{q})=\frac{v_{0}}{q^{2}+k_{\mathrm{sc}}^{2}},
$$

where $k_{\mathrm{sc}}=2 \pi / \lambda_{\mathrm{sc}}$ is the characteristic screening wave vector. We will take the two nodes to be separated by a wave vector $\mathbf{K}$. Since the screening is due to the density of electrons at a single node (characterized by $k_{F}$ ), it is reasonable to assume that $k_{\mathrm{sc}} \sim k_{F} \ll|\mathbf{K}|$.

These minimal assumptions are sufficient to estimate the rate of relaxation of charge and valley charge and therefore are the only ones necessary for the WSM. Again, the DSM case has additional subtleties, as we elucidate below.
Using Fermi's golden rule and averaging over disorder, we may estimate the relaxation time for quasiparticles on the Fermi surface as

$$
\tau_{c}^{-1}=\frac{\nu\left(\epsilon_{F}\right) n_{\mathrm{imp}}}{\pi \hbar} \int \frac{d \hat{\mathbf{k}}^{\prime}}{4 \pi}\left|v\left(k_{F} \hat{\mathbf{k}}-k_{F} \hat{\mathbf{k}}^{\prime}\right)\right|^{2},
$$

where $\nu\left(\epsilon_{F}\right)=\epsilon_{F}^{2} /\left(\hbar v_{F}\right)^{3}$ is the density of states at the Fermi level in each node and the integral is over all possible angular coordinates of the final state on the Fermi sphere. Using the form of the impurity potential described above and the fact that $k_{F} \sim k_{\mathrm{sc}}$ to approximate the angular integration, we find

$$
\tau_{c}^{-1} \approx \frac{\nu\left(\epsilon_{F}\right) n_{\mathrm{imp}}}{\pi \hbar}|v(0)|^{2} .
$$

In contrast, the charge relaxation involves a large momentum transfer as it mixes the two valleys. Parametrizing the initial and final momenta as $\mathbf{k}_{i}=\mathbf{K}+\mathbf{k}$ and $\mathbf{k}_{f}=-\mathbf{K}+\mathbf{k}^{\prime}$, we have

$$
\tau_{v}^{-1}=\frac{\nu\left(\epsilon_{F}\right) n_{\mathrm{imp}}}{\pi \hbar} \int \frac{d \hat{\mathbf{k}}^{\prime}}{4 \pi}\left|v\left[2 \mathbf{K}+k_{F}\left(\hat{\mathbf{k}}-\hat{\mathbf{k}}^{\prime}\right)\right]\right|^{2} .
$$

Since we have $k_{F} \ll|\mathbf{K}|$, it is reasonable to approximate this via

$$
\tau_{v}^{-1} \approx \frac{\nu\left(\epsilon_{F}\right) n_{\mathrm{imp}}}{\pi \hbar}|v(2 \mathbf{K})|^{2} .
$$

Therefore, we see that the ratio of the charge-and valleyrelaxation times is given by

$$
\frac{\tau_{c}}{\tau_{v}} \approx \frac{|v(2 \mathbf{K})|^{2}}{|v(0)|^{2}} \approx\left(\frac{k_{\mathrm{sc}}}{2 K}\right)^{4} \sim\left(\frac{k_{F}}{2 K}\right)^{4},
$$

where we use $|\mathbf{K}| \sim 1 / a \gg k_{F}$, with $a$ the lattice spacing. Thus, as long as the doping of a node, parametrized by $k_{F}$, is small compared to the nodal separation-which is the criterion that the nodes are clearly resolved - then the charge relaxation occurs on a parametrically shorter time scale than the intervalley scattering. Note that we have been a little cavalier in computing the relaxation rate rather than the transport lifetime (which differs by angular factors in the integration over final momenta), but in the limits of interest to us, this distinction is negligible.

\section{B. Isospin mixing and relaxation in the DSM}

We now discuss the extension of our model of disorder to the DSM, where in addition to the charge- and valleyrelaxation rates above, a crucial new quantity must be determined: the rate of isospin relaxation at a single Dirac point, due to mixing between the two Weyl nodes. Such mixing occurs both due to weak chirality-mixing 
perturbations as well as impurity scattering; we denote the relaxation rate due to the latter by $\tau_{i}^{-1}$ (see Fig. 4). We must also make some further assumptions about the disorder. In order that our model serves as a reasonable one for estimating the isospin relaxation in the DSM, we require that the characteristic length scale of the potential $v(r)$ (parametrized, for instance, by the impurity-screening length) is large compared to that of the crystalline unit cell, so that the precise position of the impurity within the unit cell is unimportant [36]. We defer a detailed treatment of disorder in the DSM to future work [37] and, for now, simply sketch the argument for why the rate for isospinchanging scattering processes is small. To do so, we must delve into a few more details of the DSM than we have thus far.

The simplest model [8,9] of DSMs, which applies to both the cases of experimental interest, is to consider $S$ - and $P$-band electrons with strong spin-orbit coupling. After incorporating the crystal field splittings allowed by the given point-group symmetry, one obtains a minimal fourband $\mathbf{k} \cdot \mathbf{p}$ Hamiltonian describing the $\left|S_{1 / 2}, \pm(1 / 2)\right\rangle$ and $\left|P_{3 / 2}, \pm(3 / 2)\right\rangle$ bands; the four remaining bands mix and gap away from the Fermi level. Here, we have chosen the axis of quantization of angular momentum to coincide with that of a crystalline point-group rotation. These bands interchange their valence or conduction character as one moves along the $\Gamma Q$ line in momentum space. Here, we denote by $Q$ the point on the zone boundary through which the rotation axis passes; in the standard Brillouin-zone labeling convention, $Q=A$ for $\mathrm{Na}_{3} \mathrm{Bi}$, which has a hexagonal space group $P 6_{3} / m m c$, and $Q=Z$ for $\mathrm{Cd}_{3} \mathrm{As}_{2}$, which has a tetragonal space group $P 4_{2} / n m c$. (In both cases, we label the axis of symmetry $k_{z}$.) Owing to the point-group symmetry along the $\Gamma Q$ line, the resulting band crossing is stable. As we move away from the $\Gamma Q$ line, the $\left|S_{1 / 2},(1 / 2)\right\rangle$ and $\left|P_{3 / 2},(3 / 2)\right\rangle$ split linearly, as do the $\left|S_{1 / 2},-(1 / 2)\right\rangle$ and $\left|P_{3 / 2},-(3 / 2)\right\rangle$, but matrix elements between these pairs are quadratic in the momentum measured from the node. A simple $\mathbf{k} \cdot \mathbf{p}$ matrix describing a single Dirac point that incorporates these symmetries is therefore [38]

$$
\hat{H}(\mathbf{k})=\left(\begin{array}{cccc}
v_{F} k_{z} & v_{F} k_{+} & 0 & \beta k_{-}^{2} \\
v_{F} k_{-} & -v_{F} k_{z} & \beta k_{-}^{2} & 0 \\
0 & \beta k_{+}^{2} & v_{F} k_{z} & -v_{F} k_{-} \\
\beta k_{+}^{2} & 0 & -v_{F} k_{+} & -v_{F} k_{z}
\end{array}\right),
$$

where we have defined $k_{ \pm}=k_{x} \pm i k_{y}$, assumed an isotropic dispersion with $\hbar=1$, and expanded about the nodal point, assumed to be $(0,0, K)$. As we see, in the absence of the quadratic curvature terms (i.e., when $\beta=0$ ), the Dirac point can be decomposed into two independent Weyl nodes of opposite chirality [39]. In the absence of this term, rotationally invariant impurities cannot scatter between the nodes. Thus, any mixing between the two isospins depends on $\beta$. Transforming to the eigenbasis of the $\beta=0$ Hamiltonian, we find

$$
\hat{U}_{\mathbf{k}} \hat{H}(\mathbf{k}) \hat{U}_{\mathbf{k}}^{-1}=\left(\begin{array}{cccc}
v_{F} k & 0 & 0 & \beta k_{-}^{2} \\
0 & -v_{F} k & \beta k_{-}^{2} & 0 \\
0 & \beta k_{+}^{2} & v_{F} k & 0 \\
\beta k_{+}^{2} & 0 & 0 & -v_{F} k
\end{array}\right)
$$

We see from Eq. (22) that the only mixing is between electronlike (holelike) states of isospin-up and holelike (electronlike) states of isospin-down. Taking $\epsilon_{F}>0$ using first-order perturbation theory in $\beta$, we find that the curvature-corrected eigenstates at the Fermi level mix the chiralities

$$
\begin{aligned}
|\mathbf{k},+, \uparrow\rangle_{c} & \approx|\mathbf{k},+, \uparrow\rangle+\frac{\beta k}{2 v_{F}} \sin ^{2} \theta_{\mathbf{k}} e^{-2 i \phi_{\mathbf{k}}}|\mathbf{k},-, \downarrow\rangle, \\
|\mathbf{k},+, \downarrow\rangle_{c} & \approx|\mathbf{k},+, \downarrow\rangle+\frac{\beta k}{2 v_{F}} \sin ^{2} \theta_{\mathbf{k}} e^{+2 i \phi_{\mathbf{k}}}|\mathbf{k},-, \uparrow\rangle,
\end{aligned}
$$

where we label the two chiralities with $\uparrow$ and $\downarrow$, the electron-hole nature is indicated by the \pm label, and we assume that $|\mathbf{k}|=k_{F}$. In order that we may treat the electronic levels from the two Weyl nodes at a single Dirac point as approximate chirality eigenstates, we must demand that $\beta k_{F} / 2 v_{F} \ll 1$. We note that a useful proxy for this assumption is that it breaks down at doping levels where the energy bands show significant deviation from linear behavior.

We now turn to an estimate of the relaxation time due to impurity scattering. With the assumption of $s$-wave impurities made above, it is straightforward to estimate the scattering rate due to impurities; we find, after a few elementary manipulations and upon disorder averaging, that it is given by

$$
\begin{aligned}
\tau_{i}^{-1}= & \frac{\nu\left(\epsilon_{F}\right) n_{\text {imp }}}{\pi \hbar} \int \frac{d \hat{\mathbf{k}}^{\prime}}{4 \pi}\left|v\left(k_{F} \hat{\mathbf{k}}-k_{F} \hat{\mathbf{k}}^{\prime}\right)\right|^{2} \\
& \times\left.\left.\right|_{c}\left\langle k_{F} \hat{\mathbf{k}},+, \uparrow \mid k_{F} \hat{\mathbf{k}}^{\prime},+, \downarrow\right\rangle_{c}\right|^{2} .
\end{aligned}
$$

Owing to the highly anisotropic nature of the matrix element, performing the angular integration in Eq. (24) is fairly complicated in the general case. However, for our purposes, it suffices to estimate an upper bound on $\tau_{i}^{-1}$. To that end, we observe that any angular dependence of the integrand can be ignored (as these only correct the numerical prefactors) and that from Eq. (23), the matrix element in Eq. (24) is proportional to $\beta k_{F} / v_{F}$. Thus, we find that a rough estimate of the impurity-induced isospinrelaxation rate is 


$$
\tau_{i}^{-1} \approx \frac{\nu\left(\epsilon_{F}\right) n_{\mathrm{imp}}}{\pi \hbar}|v(0)|^{2}\left(\frac{\beta k_{F}}{2 v_{F}}\right)^{2}=\tau_{c}^{-1}\left(\frac{\beta k_{F}}{2 v_{F}}\right)^{2} .
$$

It may be instructive to readers to note that this isospinrelaxation process bears a mathematical resemblance to the Elliot-Yafet mechanism of spin relaxation in weakly spinorbit-coupled semiconductors. We see that once again, the criterion for this rate to be small is to require that the curvature correction to the dispersion is negligible at the relevant Fermi energy.

\section{MATERIAL CANDIDATES}

\section{A. Weyl semimetals}

To date, incontrovertible evidence that any material is in the WSM phase is lacking, although the experimental situation of transport measurements in the pyrochlore iridates [40] is encouraging, particularly in $\mathrm{Eu}_{2} \mathrm{Ir}_{2} \mathrm{O}_{7}$ under pressure [41]. In addition, other materials have been suggested as WSM candidates [42-46], and theoretical proposals to engineer Weyl nodes in topological-insulator or normal-insulator heterostructures [47-49] have appeared. In the absence of an explicit realization, estimating actual values of experimental parameters is challenging. While we will provide more estimates with more experimental input in the more immediately compelling case of the DSM below, for now, we make some very general estimates that should be broadly applicable to a variety of WSM candidates.

To that end, we note that the doping level $x$ in a WSM can be estimated by counting the fraction of the Brillouinzone (BZ) volume occupied by the Fermi sphere:

$$
x=\frac{2 \times \frac{4 \pi k_{F}^{3}}{3}}{\left(2 K_{b}\right)^{3}} \approx\left(\frac{k_{F}}{K_{b}}\right)^{3},
$$

where $K_{b}$ is the momentum scale of the $\mathrm{BZ}$ and we have assumed that there are only two nodes. If we assume that $K \sim K_{b} / 2$, we find using Eqs. (20) and (26) that

$$
\frac{\tau_{c}}{\tau_{v}} \sim\left(\frac{k_{F}}{2 K}\right)^{4} \sim x^{4 / 3}
$$

Assuming a doping level of $1 \%$, we find $\tau_{v} \sim 500 \tau_{c}$. Taking a conservative estimate for the mean-free path $\ell=$ $v_{F} \tau_{c} \sim 10 \mathrm{~nm}$, we find that the valley-relaxation length $\ell_{v}$ is of the order of a few microns. We therefore see that it is not too unreasonable to expect that high-mobility samples-where the mean-free path can exceed our quite conservative estimate-may well exhibit significant, anomaly-induced nonlocal resistance over scales where it is possible to distinguish this nonlocal signature from Ohmic conductivity.

\section{B. Dirac semimetals}

In contrast to the Weyl case where there is, as yet, a convincing experimental realization, there are two promising materials that appear to exhibit Dirac semimetallic behavior in three dimensions. Following predictions from densityfunctional-theory calculations, photoemission and magnetotransport studies of the three-dimensional materials $\mathrm{Na}_{3} \mathrm{Bi}$ and $\mathrm{Cd}_{3} \mathrm{As}_{2}$ strongly suggest the presence of bulk Dirac points in these materials.

Of these, the crystal structure of $\mathrm{Na}_{3} \mathrm{Bi}$ is significantly simpler and has the added benefit of preserving inversion symmetry. In contrast, $\mathrm{Cd}_{3} \mathrm{As}_{2}$ has an 80 -site unit cell in its inversion-breaking low-energy crystal structure, complicating our assumption that we can ignore the exact position of the impurity within the unit cell and invalidating the neglect of inversion-symmetry-breaking terms that can mix chiralities at $O(k)$ rather than $O\left(k^{2}\right)$. A more careful treatment of disorder than that given in the preceding section is therefore necessary. In light of these issues, we will focus on providing estimates for various relaxation scales in the case of $\mathrm{Na}_{3} \mathrm{Bi}$.

From the photoemission data $[11,14]$ on $\mathrm{Na}_{3} \mathrm{Bi}$, we estimate that the Fermi energy (measured relative to the node) is around $\epsilon_{F} \lesssim 0.02 \mathrm{eV}$. Note that $\epsilon_{F}$ can be adjusted over a range of about $0.1 \mathrm{eV}$ by doping with potassium [11]. We approximate the Fermi velocity by $v_{F} \sim 1 \mathrm{eV}-\AA$ and ignore the anisotropy in the node dispersion to obtain a characteristic Fermi wave vector $k_{F} \sim 0.02 \AA$. Using these values in conjunction with the measured momentum-space separation of the nodes [14] $2 K \sim 0.2 \AA$ and Eq. (20), we find

$$
\frac{\tau_{c}}{\tau_{v}} \sim\left(\frac{k_{F}}{2 K}\right)^{4} \sim 10^{-4} .
$$

Thus, even the conservative estimate of the electronic mean-free path such as that used above for the WSM yields a valley-relaxation length $\ell_{v}$ of $100 \mu \mathrm{m}$. It seems reasonable to expect that various approximations (such as the neglect of anisotropy in the Fermi velocity) will only affect this estimate weakly and that $\ell_{v}$ of the order of tens of microns should be quite feasible, as in the WSM example above.

The curvature term is somewhat trickier to estimate. If we assume that the curvature emerges due to an underlying parabolic dispersion $\epsilon \sim k^{2} / 2 m^{*}$ to make a rough guess for $\beta \sim 1 / 2 m^{*} \approx\left(m / m^{*}\right) \times 3.8 \mathrm{eV}-\AA^{2}$, we can estimate

$$
\frac{\beta k_{F}}{2 v_{F}} \sim 0.04\left(\frac{m}{m^{*}}\right) .
$$

Since we anticipate $m^{*} \gtrsim m$, we see that the curvature correction is relatively small; if we set $m=m^{*}$ and simply use Eq. (26) to estimate the isospin-relaxation time, then we find 


$$
\tau_{i} \sim 10^{3} \tau_{c}
$$

which, using our estimate for the mean-free path, yields a relaxation length of around $10 \mu \mathrm{m}$. As in the Weyl case, it is reasonable to expect that the anomaly-induced nonlocality can be clearly distinguished from Ohmic effects in samples of reasonably high mobility.

\section{DISCUSSION}

We have suggested a route to studying the chiral anomaly in three-dimensional topological semimetals by using it to produce and detect valley imbalance and using the slow relaxation of the latter to produce nonlocal voltage drops, which can be distinguished from more conventional Ohmic effects. Additionally, the nonlocal response is strongly dependent on the direction of applied magnetic fields, providing a means to verify its origin in the chiral anomaly. We have tried to provide the simplest description of the nonlocal response: We have assumed that the contacts dominate the relaxation at the leads and thus the process of imbalance generation and detection, and that intervalley scattering only limits the diffusion of valley imbalance away from the contacts. Furthermore, we took the contacts to be nonideal, since metallic contacts severely constrain the generation of valley imbalance in the simple geometry proposed here. In spite of these restrictions, we find a nonlocal response that depends predominantly on parameters that can be tuned independently of the material, and no fundamental limit on the nonlocal response is apparent.

To emphasize that the use of tunneling contacts, while important in our geometry, is not fundamental to the nonlocal transport, we note that an alternative approach would be to utilize the so-called " $H$ geometry" (Fig. 5), frequently employed in spintronics. Here, two massive parts of the sample (generator $G$ and detector $D$ ) are connected by a narrow bridge of length $L \ll \ell_{v}$. Both massive parts are subject to local magnetic fields $B_{g}$ and $B_{d}$. In addition, current is driven through region $G$, which leads to the generation of valley imbalance. Thus, this region acts as a "valley battery." The valley imbalance diffuses over the bridge to region $D$, where once again, the chiral anomaly gives rise to a measurable voltage drop, similar to the one studied above. The $H$ geometry may offer some practical advantages for producing and measuring valley currents in WSMs. In particular, this geometry increases the effective value of $d$ in the generation region, in turn increasing the source-drain-diffusion time, and thereby reducing $\Gamma_{\text {leads, }}$, and allows the generation of a sizable valley imbalance, limited only by intervalley impurity scattering, even with good metallic contacts.

We also constructed a simple model of scattering from screened impurities, within which we were able to provide estimates for the relevant relaxation scales applicable to the experimentally relevant case of the DSM material

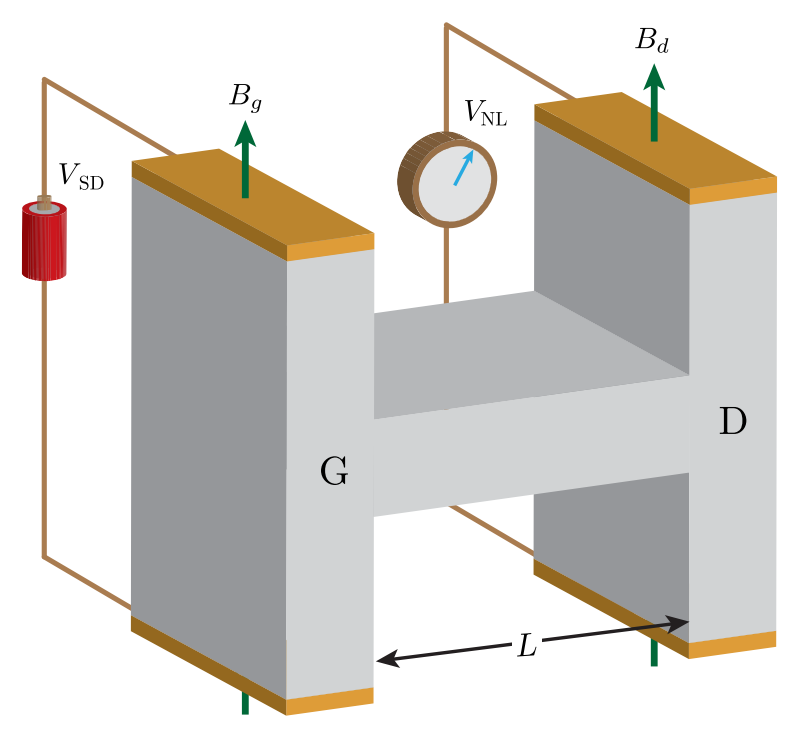

FIG. 5. $H$ geometry. An alternative setup in which the generation and detection regions are massive, so that valley relaxation at the leads is diminished, making metallic contacts feasible.

$\mathrm{Na}_{3} \mathrm{Bi}$. The scales obtained are within current experimental capabilities and suggest that nonlocal magnetotransport measurements on high-mobility samples of this material may be key to unraveling its topological nature.

In closing, we observe that since the chiral anomaly is the distinctive topological EM response of a topological semimetal, the fact that its effects have such a dramatic manifestation in relatively simple transport measurements suggests that they may be useful in the search for both Weyl and Dirac materials in three dimensions. Similar nonlocal probes may be relevant to other cases in which topological features exist even in systems that lack a bulk gap.

\section{ACKNOWLEDGEMENTS}

We thank L. Balents, J. H. Bardarson, A. Burkov, Y.-B. Kim, R. Ilan, N. P. Ong, and B. Z. Spivak for useful discussions on transport; F. de Juan, I. Kimchi, P. Dumitrescu, N.P. Ong, and especially A. Potter for conversations on Dirac semimetals; and an anonymous referee for comments on an earlier version of this manuscript. This work was supported in part by the Simons Foundation (S. A. P.); the NSF under Grant No. PHYS1066293 and the hospitality of the Aspen Center for Physics (S. A. P. and D. A. P.); the Director, Office of Science, Office of Basic Energy Sciences, Materials Sciences and Engineering Division, of the U.S. Department of Energy under Contract No. DE-AC02$05 \mathrm{CH} 11231$ (A. V.); and the Institute for Quantum Information and Matter, a NSF Physics Frontiers Center, with support of the Gordon and Betty Moore Foundation through Grant No. GBMF1250 (D. A. P.). 
[1] X. Wan, A. M. Turner, A. Vishwanath, and S. Y. Savrasov, Topological Semimetal and Fermi-Arc Surface States in the Electronic Structure of Pyrochlore Iridates, Phys. Rev. B 83, 205101 (2011).

[2] C. Herring, Effect of Time-Reversal Symmetry on Energy Bands of Crystals, Phys. Rev. 52, 361 (1937).

[3] A. A. Abrikosov and S. D. Beneslavskii, Some Properties of Gapless Semiconductors of the Second Kind, J. Low Temp. Phys. 5, 141 (1971).

[4] G. E. Volovik, The Universe in a Helium Droplet (Clarendon, Oxford, England, 2003).

[5] H. B. Nielsen and M. Ninomiya, The Adler-Bell-Jackiw Anomaly and Weyl Fermions in a Crystal, Phys. Lett. 130B, 389 (1983).

[6] A. M. Turner and A. Vishwanath, Beyond Band Insulators: Topology of Semi-metals and Interacting Phases, arXiv:1301.0330.

[7] S. M. Young, S. Zaheer, J. C. Y. Teo, C. L. Kane, E. J. Mele, and A. M. Rappe, Dirac Semimetal in Three Dimensions, Phys. Rev. Lett. 108, 140405 (2012).

[8] Z. Wang, Y. Sun, X.-Q. Chen, C. Franchini, G. Xu, H. Weng, X. Dai, and Z. Fang, Dirac Semimetal and Topological Phase Transitions in $\mathrm{A}_{3} \mathrm{Bi}(A=N a, K, R b)$, Phys. Rev. B 85, 195320 (2012).

[9] Z. Wang, H. Weng, Q. Wu, X. Dai, and Z. Fang, ThreeDimensional Dirac Semimetal and Quantum Transport in $\mathrm{Cd}_{3} \mathrm{As}_{2}$, Phys. Rev. B 88, 125427 (2013).

[10] Throughout, we will be careful to distinguish between Dirac points and Weyl nodes, so that we can refer to the two Weyl nodes at a single Dirac point.

[11] Z. K. Liu, B. Zhou, Z. J. Wang, H. M. Weng, D. Prabhakaran, S.-K. Mo, Y. Zhang, Z. X. Shen, Z. Fang, X. Dai, Z. Hussain, and Y. L. Chen, Discovery of a Three-Dimensional Topological Dirac Semimetal, $\mathrm{Na}_{3} \mathrm{Bi}$, Science 343, 864 (2014).

[12] M. Neupane, S. Xu, R. Sankar, N. Alidoust, G. Bian, C. Liu, I. Belopolski, T.-R. Chang, H.-T. Jeng, H. Lin, A. Bansil, F. Chou, and M. Z. Hasan, Observation of a Topological 3D Dirac Semimetal Phase in High-Mobility $\mathrm{Cd}_{3} \mathrm{As}_{2}$ and Related Materials, arXiv:1309.7892.

[13] S. Borisenko, Q. Gibson, D. Evtushinsky, V. Zabolotnyy, B. Buechner, and R. J. Cava, Experimental Realization of a Three-Dimensional Dirac Semimetal, Phys. Rev. Lett. 113, 027603 (2014).

[14] S.-Y. Xu et al., Observation of a Bulk 3D Dirac Multiplet, Lifshitz Transition, and Nestled Spin States in $\mathrm{Na}_{3} \mathrm{Bi}$, arXiv: 1312.7624.

[15] N. P. Ong (private communication).

[16] S. L. Adler, Axial-Vector Vertex in Spinor Electrodynamics, Phys. Rev. 177, 2426 (1969).

[17] J. S. Bell and R. Jackiw, A PCAC Puzzle: $\pi_{0} \rightarrow \gamma \gamma$ in the $\sigma$-Model, Nuovo Cimento A 60, 47 (1969).

[18] D. A. Abanin, A. V. Shytov, L. S. Levitov, and B. I. Halperin, Nonlocal Charge Transport Mediated by Spin Diffusion in the Spin Hall Effect Regime, Phys. Rev. B 79, 035304 (2009).

[19] D. A. Abanin, S. V. Morozov, L. A. Ponomarenko, R. V. Gorbachev, A. S. Mayorov, M. I. Katsnelson, K. Watanabe, T. Taniguchi, K. S. Novoselov, L. S. Levitov, and A. K. Geim, Giant Nonlocality Near the Dirac Point in Graphene, Science 332, 328 (2011).
[20] D. A. Abanin, R. V. Gorbachev, K. S. Novoselov, A. K. Geim, and L.S. Levitov, Giant Spin-Hall Effect Induced by the Zeeman Interaction in Graphene, Phys. Rev. Lett. 107, 096601 (2011).

[21] J. Balakrishnan, G. Kok Wai Koon, M. Jaiswal, A. H. Castro Neto, and B. Ozyilmaz, Colossal Enhancement of SpinOrbit Coupling in Weakly Hydrogenated Graphene, Nat. Phys. 9, 284 (2013).

[22] T. D. C. Bevan, A. J. Manninen, J. B. Cook, J. R. Hook, H. E. Hall, T. Vachaspati, and G. E. Volovik, Momentum Creation by Vortices in Superfluid ${ }^{3} \mathrm{He}$ as a Model of Primordial Baryogenesis, Nature (London) 386, 689 (1997).

[23] V. Aji, Adler-Bell-Jackiw Anomaly in Weyl Semimetals: Application to Pyrochlore Iridates, Phys. Rev. B 85, 241101 (2012).

[24] D. T. Son and B. Z. Spivak, Chiral Anomaly and Classical Negative Magnetoresistance of Weyl Metals, Phys. Rev. B 88, 104412 (2013).

[25] A. A. Burkov, M. D. Hook, and L. Balents, Topological Nodal Semimetals, Phys. Rev. B 84, 235126 (2011).

[26] C.-X. Liu, P. Ye, and X.-L. Qi, Chiral Gauge Field and Axial Anomaly in a Weyl Semi-metal, Phys. Rev. B 87, 235306 (2013).

[27] K.-Y. Yang, Y.-M. Lu, and Y. Ran, Quantum Hall Effects in a Weyl Semimetal: Possible Application in Pyrochlore Iridates, Phys. Rev. B 84, 075129 (2011).

[28] For instance, for $B \neq 0$, there is always a net uniform valley current $\boldsymbol{j}^{R}-\boldsymbol{j}^{L} \propto \mathbf{B}$ even in equilibrium, but this current is unimportant to the transport calculation and we therefore ignore it.

[29] Throughout, we assume an isotropic node dispersion.

[30] D. T. Son and P. Surówka, Hydrodynamics with Triangle Anomalies, Phys. Rev. Lett. 103, 191601 (2009).

[31] Note the extra factor of $e$ for the electrical current.

[32] Although not crucial, this assumption simplifies the analysis.

[33] The precise number will depend on the sample size.

[34] A similar problem arises with spin injection with ferromagnetic leads.

[35] E. I. Rashba, Theory of Electrical Spin Injection: Tunnel Contacts as a Solution of the Conductivity Mismatch Problem, Phys. Rev. B 62, R16267 (2000).

[36] In the opposite limit, even a spherically symmetric impurity might lead to mixing between isospins, as the placement of the impurity away from the symmetry center would strongly break the crystalline point-group symmetry.

[37] S. A. Parameswaran, D. A. Pesin, D. A. Abanin, and A. Vishwanath (unpublished).

[38] Note that, for simplicity, we have ignored an inversionbreaking term that is required to properly describe cadmium arsenide; we defer a discussion of such details to future work.

[39] Observe that in contrast to the case of vacuum Dirac fermions familiar to high-energy physicists, this decomposition is unambiguous in a crystal since electrons emanating from the two Weyl points carry distinct crystal-symmetry quantum numbers; this is also why a mass term is forbidden by symmetry. 
[40] D. Yanagishima and Y. Maeno, Metal-Nonmetal Changeover in Pyrochlore Iridates, J. Phys. Soc. Jpn. 70, 2880 (2001).

[41] F. F. Tafti, J. J. Ishikawa, A. McCollam, S. Nakatsuji, and S. R. Julian, Pressure-Tuned Insulator to Metal Transition in $\mathrm{Eu}_{2} \mathrm{Ir}_{2} \mathrm{O}_{7}$, Phys. Rev. B 85, 205104 (2012).

[42] G. Xu, H. Weng, Z. Wang, X. Dai, and Z. Fang, Chern Semimetal and the Quantized Anomalous Hall Effect in $\mathrm{HgCr}_{2} \mathrm{Se}_{4}$, Phys. Rev. Lett. 107, 186806 (2011).

[43] W. Witczak-Krempa and Y. Baek Kim, Topological and Magnetic Phases of Interacting Electrons in the Pyrochlore Iridates, Phys. Rev. B 85, 045124 (2012).

[44] J. L. Mañes, Existence of Bulk Chiral Fermions and Crystal Symmetry, Phys. Rev. B 85, 155118 (2012).
[45] X. Wan, A. Vishwanath, and S. Y. Savrasov, Computational Design of Axion Insulators Based on $5 d$ Spinel Compounds, Phys. Rev. Lett. 108, 146601 (2012).

[46] Y. Chen, D. L. Bergman, and A. A. Burkov, Weyl Fermions and the Anomalous Hall Effect in Metallic Ferromagnets, Phys. Rev. B 88, 125110 (2013).

[47] A. A. Burkov and L. Balents, Weyl Semimetal in a Topological Insulator Multilayer, Phys. Rev. Lett. 107, 127205 (2011).

[48] G. B. Halász and L. Balents, Time-Reversal Invariant Realization of the Weyl Semimetal Phase, Phys. Rev. B 85, 035103 (2012).

[49] G. Y. Cho, Possible Topological Phases of Bulk Magnetically Doped $\mathrm{Bi}_{2} \mathrm{Se}_{3}$ : Turning a Topological Band Insulator into the Weyl Semimetal, arXiv:1110.1939. 